\title{
Validación del recurso solar en el Ecuador para aplicaciones de media y alta temperatura
}

\section{Validation of the solar resource in Ecuador for medium and high temperature applications}

\author{
Sandra Arla Odio \\ Melton Tapia Zurita \\ José Guasumba Codena \\ Universidad de las Fuerzas Armadas, Ecuador \\ Johanna Martínez \\ Usva Energía Cía. Ltda., Ecuador \\ Julio Asitimbay \\ Oleoducto de Crudos Pesados S. A. (OCP), Ecuador \\ Edison Tapia Zurita \\ Soluciones en estructuras metálicas (SEDEMI), Ecuador
}

Autor para correspondencia: smarla@espe.edu.ec, metapia@espe.edu.ec

Fecha de recepción: 11 de Abril de 2017 - Fecha de aceptación: 30 de Junio de 2017

Resumen: El estudio provee información real del recurso energético solar a través de mediciones de la radiación global realizadas en las estaciones meteorológicas, ubicadas a lo largo del trayecto del oleoducto de crudos pesados en 15 puntos de interés en las provincias Nueva Loja, Napo, Pichincha y Esmeraldas, el promedio de estas es comparado con los valores medios obtenidos mediante procedimientos mundialmente utilizados: observaciones por satélite, estimaciones por software y estimaciones por modelos matemáticos. Este estudio pone a disposición de los diseñadores de sistemas de energía solar en Ecuador datos confiables de la radiación solar global, de modo que se puedan dimensionar los sistemas con datos reales y además se tenga conocimiento del grado de error existente al utilizar estos procedimientos; dando a conocer las diferencias encontradas entre los valores medios de radiación determinados por modelos de simulación y de correlación con los medidos en superficie.

Palabras clave: recurso energético; radiación solar; medición

\begin{abstract}
The study provides real information on the solar energy resource through global radiation measurements carried out at meteorological stations located along the heavy oil pipeline route at 15 points of interest in the provinces Nueva Loja, Napo, Pichincha and Esmeraldas, The average of these is compared with the average values obtained through globally used procedures: satellite observations, software estimates and estimates by mathematical models. This study makes available to the designers of solar energy systems in Ecuador reliable data of the global solar radiation, so that the systems with real data can be dimensioned and also the degree of error is known when using these procedures; Revealing the differences found between the mean values of radiation determined by simulation and correlation models with those measured on the surface.
\end{abstract}

Key words: energy resource; solar radiation; measurement 


\section{Introducción}

El reto actual del Ecuador es el cambio de la matriz energética. Se han hecho esfuerzos para desarrollar aplicaciones utilizando el recurso energético solar en diversos campos, sin embargo, esta tecnología no se ha desarrollado adecuadamente, lo que ocasiona retrasos en la aplicación y baja confiabilidad de esta energía alternativa frente al consumidor final.

El gobierno es el responsable de impulsar una nueva gestión de la energía en Ecuador por medio de políticas que prioricen el aprovechamiento del recurso solar y el manejo adecuado de los combustibles fósiles. La investigación y desarrollo de las energías alternativas deben tener una secuencia estratégica en la planificación de estos vectores energéticos; no se puede innovar tecnología sin antes haber evaluado la disponibilidad y calidad del recurso con el que cuenta el país. El primer paso en el cambio y desarrollo de las energías no convencionales es la evaluación de los recursos renovables disponibles, en lo que las instituciones educativas junto a los profesionales emplazados en el área energética pueden aportar con investigaciones.

Para implementar tecnologías con aplicación de media y alta temperatura solar, es necesario contar con radiación directa de al menos 3 horas por día y valores de irradiancia en el intervalo de 500 a $1000 \mathrm{~W} / \mathrm{m}^{2}$ (Suntaxi, J, 2014).

La falta de estaciones meteorológicas en puntos estratégicos del país y la no existencia de datos climatológicos hace difícil medir el recurso solar con precisión. Con lo mencionado, en Ecuador, los análisis se realizan mediante el estudio de fuentes bibliográficas como el "Mapa bioclimático del Ecuador" de L. Cañada (Cañada, 1983) y de datos procedentes de las estaciones meteorológicas del Instituto Nacional de Meteorología e Hidrografía, además de las modelizaciones climáticas allá donde los datos reales son insuficientes, planteadas por L, Velasco en su investigación sobre la biomasa vegetal como aislamiento en climas extremos del Ecuador (Velasco Roldan, Goyos Pérez, Freire Amores , \& Ibarra, 2015).

Actualmente se dispone de valores de insolación, como los descritos en el "Atlas Solar del Ecuador con Fines de Generación Eléctrica" (2008), los cuales se basan en información correspondiente al periodo 01/01/1985 - 31/12/1991, generada por el "National Renewable Energy Laboratory" (NREL) de los Estados Unidos, a través del "Climatological Solar Radiation Model” (Consejo Nacional de Electricidad del Ecuador, 2008); y aquélla proporcionada por el INAMHI, del cual solo una de sus estaciones que actualmente observa el recurso solar, coincide con la ubicación del área de estudio. Los investigadores no cuentan con observaciones de radiación solar medidas en superficie que permitan realizar un dimensionamiento próximo a la realidad, para plantear propuestas de aplicaciones de media y alta temperatura, por lo que se presenta la necesidad de generar información confiable sobre el recurso solar (Suntaxi, J, 2014).

La dirección de la radiación solar directa cae casi perpendicular al plano horizontal en zonas cercanas a la línea equinoccial. Estudios realizados determinan que el Ecuador, por su ubicación geográfica, es considerado un área de alto potencial de radiación solar donde se estima que la radiación incidente media es de 3-4 kwh/m2/día (Orellana \& Orellana Samaniego, 2015). 
El cumplimiento del objetivo general de esta investigación facilita el desempeño de estos escenarios al determinar la radiación solar en el área aledaña a la ruta que sigue el Oleoducto de Crudos Pesados, mediante la aplicación de técnicas estadísticas para determinar los valores reales para la implementación de aplicaciones en el área o en otros puntos del Ecuador.

\section{Materiales y Métodos}

El trabajo se desarrolló a lo largo de $485 \mathrm{~km}$ lineales, partiendo de la estación terminal Amazonas ubicada a 305 m s.n.m. en la ciudad de Nueva Loja, provincia de Sucumbíos. En el trayecto, llega a la altitud máxima de 4064 m.s.n.m., sitio de la Virgen de Papallacta. La ruta atraviesa la provincia de Pichincha y en la hoya de Quito describe un arco por el norte del Distrito Metropolitano hasta cruzar la Cordillera Occidental de los Andes a 3300 m s. n. m., luego desciende por el noroccidente ecuatoriano hasta los 206 m s. n. m., sector de Punta Gorda cercano a la ciudad de Esmeraldas, provincia del mismo nombre, en donde se localiza el terminal marítimo junto al Océano Pacífico, lugar en el que concluye el oleoducto. Este atraviesa 11 cantones y 4 provincias (OCP, 2012), la figura 1 muestra el trayecto del oleoducto.

\section{La ruta que recorre el oleoducto ecuatoriano}

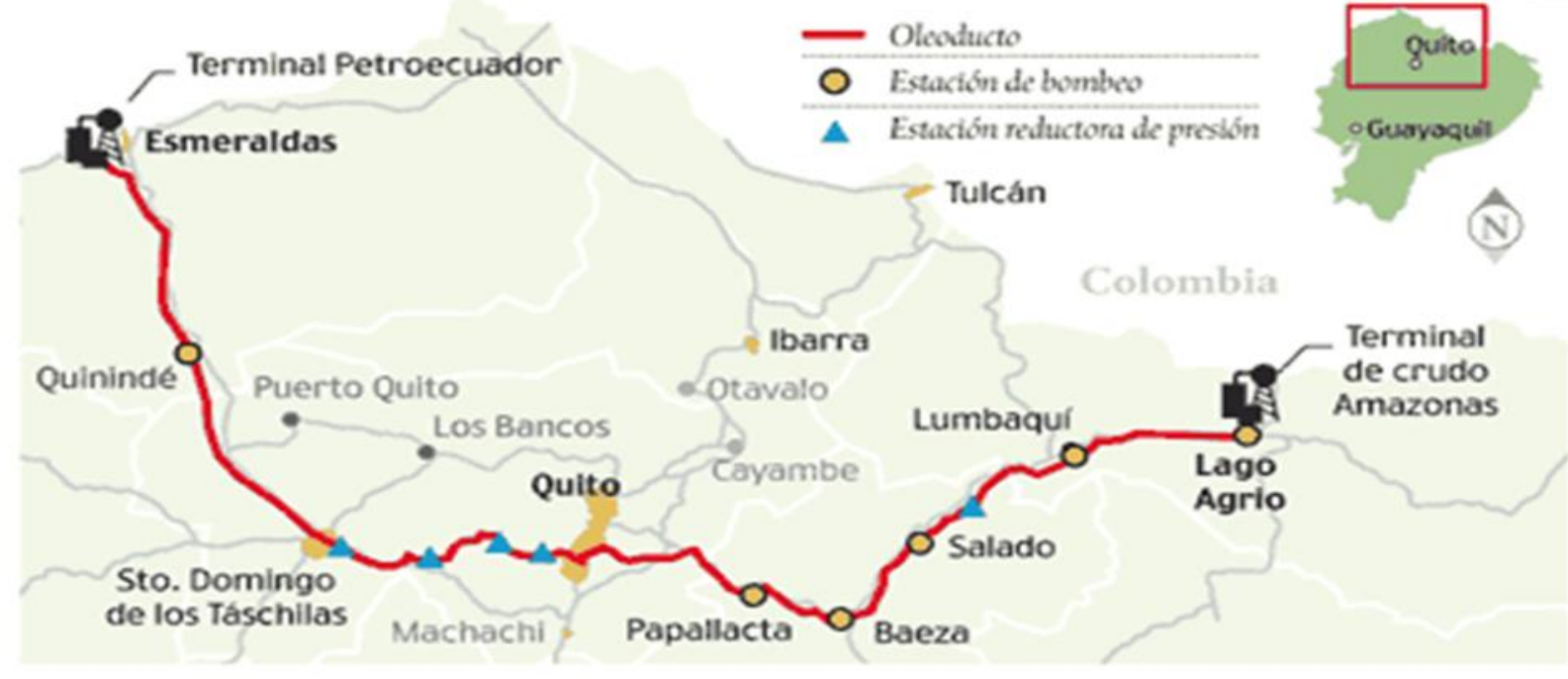

Figura 1. Trayectoria del OCP como área de investigación

\section{Procesamiento de Datos}

El valor medio de radiación solar global, calculado a partir de las observaciones en superficie, se contrasta con los valores obtenidos a través de distintos procedimientos para la determinación de la radiación, como observaciones realizadas con satélites (NASA), mediante programas informáticos (CENSOLAR) y estimaciones por modelo matemático (ANGSTRÖM \& PAGE).

\section{Observaciones por Satélite}


Las imágenes satelitales corresponden al tipo mapa de bits. Estas imágenes tienen como unidad principal el pixel (unidad mínima que compone una imagen bitmap). Cada píxel contiene información de ubicación y color y la particularidad de no poseer un tamaño definido, el mismo va a estar determinado por la resolución de la imagen. La resolución de una imagen mapa de bits está dada por la cantidad de píxeles concentrados por unidad de medida, en una relación directa que determina que a mayor concentración mejor calidad de la imagen y a la vez mayor tamaño (peso) del archivo (OCP, 2012).

En el caso concreto de las imágenes de satélite, el término resolución está principalmente relacionado con la definición, o metros (como unidad principal dentro del píxel) que contiene cada píxel. Entonces, en las imágenes de satélite, los píxeles corresponden a un área de terreno estudiada en un instante determinado por el radiómetro, en el cual se mantiene el ángulo de apertura o de visión. El terreno abarcado por un píxel, en el Ecuador es menor en el punto del subsatélite y conforme se aleja de este punto el área contenida en un píxel va aumentando. Esto hace que la resolución no sea constante en los píxeles de la imagen, si bien hay un único valor asociado a cada píxel. Los pixeles concretos de una imagen satelital constituyen tonos de grises que son en realidad una representación de valores en escala de 0 - 255 (OpenCourseWare, UNIA, s.f.)

Los valores de la radiación son recibidos por el satélite y luego modificados a niveles de brillo para presentarse en las posiciones relativas a través de dispositivos fotográficos o electrónicos apropiados como monitores, por ejemplo. Las imágenes captadas se clasifican en: imágenes visibles, e imágenes en infrarrojo. Los satélites permiten observar en áreas de difícil acceso como en océanos o sitios remotos. La información obtenida en superficie es muy bien complementada con la observación satelital (Nuñez \& Gentile, 2004).

Según la NASA, la media mensual de radiación solar en [kwh/m2/día] que incide sobre una superficie horizontal de la Tierra en un mes específico, es igual al promedio para ese mes en un período de 22 años y los valores promedio mensuales se evalúan como la media de valores cada 3 horas para un determinado mes (Suntaxi, J, 2014). Los valores Mínimo y Máximo de radiación solar global de un determinado mes indican la diferencia porcentual entre el año que tiene la menor (mínimo) o mayor (máximo) insolación mensual promedio en los 22 años de análisis. La figura 2 muestra los valores calculados de la media anual como valor representativo de radiación solar global para los poblados en donde se ubican las Estaciones Meteorológicas del OCP. 


\begin{tabular}{|c|c|c|c|c|c|c|c|c|c|c|c|c|c|c|}
\hline & \multirow{2}{*}{ Nisa } & \multirow[b]{3}{*}{$\begin{array}{c}\text { ANUAL } \\
\text { AVERAGE }\end{array}$} & \multicolumn{12}{|c|}{ MONTHLY AVERAG EDINSOLATION INCIDENT ON HORIZONTAL SURFACE } \\
\hline & & & \multicolumn{12}{|c|}{$\mathrm{KWh} / \mathrm{m} 2 / \mathrm{day}$} \\
\hline & CANTON & & JAN & FEB & MAR & APR & MAY & JUN & JUL & AUG & SEP & OCT & NOV & DEC \\
\hline Nueva Loja & Lago Agrio & 3,76 & 3,94 & 3,68 & 3,5 & 3,63 & 3,58 & 3,38 & 3,4 & 3,63 & 4,09 & 4,29 & 4,11 & 3,93 \\
\hline Lumbaqui & $\begin{array}{c}\text { Gonzalo } \\
\text { Pizarro }\end{array}$ & 4,24 & 4,13 & 4,08 & 4,2 & 4,13 & 4,13 & 4,2 & 4,29 & 4,38 & 4,4 & 4,42 & 4,34 & 4,15 \\
\hline \begin{tabular}{c|} 
El \\
Reventador
\end{tabular} & $\begin{array}{l}\text { Gonz } \\
\text { Piza }\end{array}$ & 3,88 & 3,86 & 3,66 & 3,64 & 3,71 & 3,75 & 3,76 & 3,82 & 4,04 & 4,11 & 4,11 & 4,15 & 3,95 \\
\hline \begin{tabular}{c|c|} 
El \\
Reventador \\
\end{tabular} & $\begin{array}{l}\text { Gonzalo } \\
\text { Pizarro }\end{array}$ & 3,88 &, 85 & 3,65 & 3,64 & 3,71 & 3,75 & 3,76 & 3,82 & 4,04 & 4,11 & 4,11 & 4,15 & 3,95 \\
\hline $\begin{array}{c}\text { Gonzalo Diaz } \\
\text { de Pineda } \\
\end{array}$ & El Chaco & 3,88 & 3,85 & 3,88 & 3,86 & 3,85 & 3,86 & 3,86 & 3,86 & 3,86 & 3,86 & 3,86 & 3,86 & 3,86 \\
\hline Santa Rosa & El Chaco & 3,88 & 3,85 & 3,66 & 3,64 & 3,71 & 3,75 & 3,76 & 3,82 & 4,04 & 4,11 & 4,11 & 4,15 & 3,95 \\
\hline $\begin{array}{c}\text { San } \\
\text { Francisco }\end{array}$ & Quijos & 3,88 & 3,86 & 3,86 & 3,86 & 3,86 & 3,66 & 3,86 & 3,88 & 3,86 & 3,86 & 3,86 & 3,86 & 3,86 \\
\hline Papallacta & Quijos & 4,25 & 4,13 & 4,34 & 4,55 & 4,33 & 4,12 & 4,01 & 4,26 & 4,46 & 4,26 & 4,24 & 4,3 & 3,98 \\
\hline Yaruqui & Quito & 4,25 & 4,13 & 4,34 & 4,55 & 4,33 & 4,12 & 4,01 & 4,26 & 4,46 & 4,26 & 4,24 & 4,3 & 3,98 \\
\hline Nono & Quito & 4,25 & 4,13 & 3,95 & 4,55 & 4,33 & 4,12 & 4,01 & 4,26 & 4,46 & 4,26 & 4,24 & 4,3 & 3,98 \\
\hline $\begin{array}{c}\text { Pedro } \\
\text { Vicente }\end{array}$ & $\begin{array}{l}\text { Pedro } \\
\text { Vicente }\end{array}$ & 3,96 & 3,95 & 4,43 & 3,96 & 3,95 & 3,95 & 3,96 & 3,95 & 3,96 & 3,95 & 3,96 & 3,96 & 3,95 \\
\hline Puertc & $\begin{array}{c}\text { Puerto } \\
\text { Quito }\end{array}$ & 4,2 & 4,24 & 4,43 & 5,00 & 4,8 & 4,22 & 3,73 & 3,87 & 4,05 & 4,21 & 3,98 & 3,81 & 4,07 \\
\hline Rosa Zarate & Quininde & 4,2 & 4,24 & 4,43 & 5,00 & 4,8 & 4,22 & 3,73 & 3,87 & 4,05 & 4,21 & 3,98 & 3,81 & 4,07 \\
\hline Viche & Quininde & 4,2 & 4,24 & 4,43 & 5,00 & 4,8 & 4,22 & 3,73 & 3,87 & 4,05 & 4,21 & 3,98 & 3,81 & 4,07 \\
\hline 'uelta Larga & Esmeraldas & 4,2 & 4,24 & 4,43 & 5,00 & 4,8 & 4,22 & 3,73 & 3,87 & 4,05 & 4,21 & 3,98 & 3,81 & 4,07 \\
\hline
\end{tabular}

Figura 2. NASA - Promedios mensual y anual de radiación solar para los sitios en donde se ubican las estaciones meteorológicas del OCP

\section{Estimaciones por Software}

El Centro de Estudios de la Energía Solar (CENSOLAR) es la entidad que forma especialistas en aspectos de energía solar y difunde las tecnologías para la utilización de la misma. La herramienta que estima la radiación solar es CENSOL que es un software desarrollado por CENSOLAR. (Calvache \& Verdugo, 2011).

Los promedios calculados por CENSOLAR fueron analizados estadísticamente para obtener la Media anual como valor representativo de radiación solar global para los poblados en donde se ubican las Estaciones Meteorológicas del OCP, según se describe en la figura 3. 


\begin{tabular}{|c|c|c|c|c|c|c|c|c|c|c|c|c|c|c|}
\hline \multirow[b]{3}{*}{ UIA } & 大 & & \multicolumn{12}{|c|}{ MONTHLY AVERAG ED INSOLATION INCIDENT ON HORIZONTAL SURFACE } \\
\hline & \multicolumn{2}{|l|}{ Censolar } & \multicolumn{12}{|c|}{$\mathrm{KWh} / \mathrm{m} 2 /$ day } \\
\hline & CANTON & AVERAGE & JAN & FEB & MAR & APR & MAY & JUN & JUL & AUG & SEP & OCT & NOV & DEC \\
\hline Nueva Loja & Lago Agrio & 3,64 & 3,5 & 3,83 & 4,14 & 4,05 & 3,95 & 3,33 & 3,7 & 3,56 & 3,39 & 3,47 & 3,39 & 3,33 \\
\hline Lumbaqui & $\begin{array}{l}\text { Gonzalo } \\
\text { Pizarro }\end{array}$ & 3,64 & 3,5 & 3,83 & 4,14 & 4,05 & 3,95 & 3,33 & 3,7 & 3,56 & 3,39 & 3,47 & 3,39 & 3,33 \\
\hline $\begin{array}{c}\text { El } \\
\text { Reventador }\end{array}$ & $\begin{array}{l}\text { Gonzalo } \\
\text { Pizarro }\end{array}$ & 4,8 & 4,57 & 4,61 & 4,78 & 4,75 & 4,78 & 4,45 & 5,2 & 5,06 & 4,78 & 4,92 & 4,81 & 4,76 \\
\hline $\begin{array}{c}\text { El } \\
\text { Reventador }\end{array}$ & $\begin{array}{c}\text { Gonzalo } \\
\text { Pizarro }\end{array}$ & 4,99 & 4,95 & 4,64 & 4,78 & 4,53 & 4,83 & 4,7 & 5,53 & 5,47 & 4,89 & 5,25 & 5,14 & 5,14 \\
\hline $\begin{array}{c}\text { Gonzalo Diaz } \\
\text { de Pineda }\end{array}$ & El Chaco & 3,44 & 3,14 & 3,14 & 3,14 & 3,14 & 3,14 & 3,14 & 3,14 & 3,14 & 3,14 & 3,14 & 3,14 & 3,14 \\
\hline Santa Rosa & El Chaco & 3,44 & 3,14 & 3,5 & 3,83 & 3,75 & 3,5 & 3,11 & 3,47 & 3,5 & 3,39 & 3,5 & 3,33 & 3,25 \\
\hline $\begin{array}{c}\text { San } \\
\text { Francisco }\end{array}$ & Quijos & 4,01 & 3,83 & 3,83 & 3,83 & 3,83 & 3,83 & 3,83 & 3,83 & 3,83 & 3,83 & 3,83 & 3,83 & 3,83 \\
\hline Papallacta & Quijos & 4,01 & 3,83 & 4,53 & 3,53 & 4,14 & 4,14 & 3,39 & 3,83 & 3,83 & 3,78 & 4,33 & 4,25 & 4,55 \\
\hline Yaruqui & Quito & 43,44 & 3,14 & 3,5 & 3,63 & 3,75 & 3,5 & 3,11 & 3,47 & 3,5 & 3,39 & 3,5 & 3,33 & 3,25 \\
\hline Nono & Quito & 4,99 & 4,95 & 4,64 & 4,78 & 4,53 & 4,83 & 4,7 & 5,53 & 5,47 & 4,89 & 5,25 & 5,14 & 5,14 \\
\hline $\begin{array}{c}\text { Pedro } \\
\text { Vicente }\end{array}$ & $\begin{array}{c}\text { Pedro } \\
\text { Vicente }\end{array}$ & 3,64 & 3,5 & 3,5 & 3,5 & 3,5 & 3,5 & 3,5 & 3,5 & 3,5 & 3,5 & 3,5 & 3,5 & 3,5 \\
\hline Puerto Quito & $\begin{array}{l}\text { Puerto } \\
\text { Quito }\end{array}$ & 4,78 & 4,72 & 4,92 & 4,56 & 4,25 & 4,47 & 4,61 & 4,97 & 5,08 & 5 & 5,03 & 4,92 & 4,81 \\
\hline Rosa Zarate & Quininde & 4,61 & 4,5 & 4,39 & 4,28 & 4,28 & 4,47 & 4,28 & 4,83 & 4,95 & 4,58 & 4,67 & 4,45 & 4,42 \\
\hline Viche & Quininde & 4,61 & 4,5 & 4,39 & 4,28 & 4,28 & 4,47 & 4,28 & 4,83 & 4,95 & 4,58 & 4,67 & 4,45 & 4,42 \\
\hline Vuelta Larga & Esmeraldas & 3,86 & 3,64 & 4,00 & 4,45 & 4,28 & 4,00 & 3,56 & 3,83 & 3,78 & 3,81 & 3,89 & 3,67 & 3,47 \\
\hline
\end{tabular}

Figura 3. CENSOLAR - Promedios mensual y anual de radiación solar para las latitudes más cercanas a las estaciones meteorológicas del OCP

\section{Estimaciones por Modelo Matemático}

El modelo matemático aplicado para conocer el recurso solar corresponde a la correlación de Angström - Prescott, modificada por Page (Prando, 2014).

Mediante este procedimiento se utilizan ecuaciones para determinar la radiación extraterrestre (He); esto se lo hace de acuerdo a la ubicación geográfica del punto en estudio y el "día tipo" para cada mes de un año. Las formulas siguientes se aplican para calcular la radiación sobre superficie horizontal (Ho). (Nuñez \& Gentile, 2004)

$\delta=23.45 \sin \left(360 \frac{284+z}{365}\right)$
$\cos h_{s}=-\tan \emptyset \tan \delta$
$N=\frac{2}{15}(\operatorname{arcos}(-\tan \emptyset \tan \delta))$

$H c=\frac{24}{\pi} I_{s c}\left(\left[+0.003 \cos \left(\frac{360 z}{365}\right)\right]\left[\cos \emptyset \cos \delta \sin h_{s}+\frac{2 \pi h_{s}}{360} \sin \emptyset \sin \delta\right]\right)$

Donde: 
$H_{c}:$ Radiacion extraterrestre en $\mathrm{KJ} / \mathrm{m}^{2}$

I_sc: Constante solar de 1360 julios/(s.m² )

Z: número de día del año

$\emptyset$ : latitud

$\delta$ :declinación

H_o:Radiacion global diaria,media

Mensual sobre una superficie situada bajo la atmosfera.

n_real: Horas de insolación

a y b: coeficientes que dependen del tipo de clima.

$\begin{array}{lcc}\text { Región } & \mathrm{a} & \mathrm{b} \\ \text { Sierra } & 0.25 & 0.45 \\ \text { Costa } & 0.28 & 0.54\end{array}$

A través del modelo matemático de Angström-Page fueron obtenidos promedios, los cuales fueron analizados estadísticamente para obtener la media anual como valor representativo de radiación solar global para los poblados en donde se ubican las estaciones meteorológicas del OCP, según se describe en la figura 4.

\begin{tabular}{|c|c|c|c|c|c|c|c|c|c|c|c|c|c|c|}
\hline \multirow[b]{2}{*}{ PARROQUIA } & \multirow[b]{2}{*}{ CANTON } & \multirow[b]{2}{*}{$\begin{array}{c}\text { ANUAL } \\
\text { AVERAGE }\end{array}$} & \multicolumn{12}{|c|}{ ANGSTROM - PAGE [kWh/m2/day] } \\
\hline & & & JAN & FEB & MAR & APR & MAY & JUN & JUL & AUG & SEP & OCT & NOV & DEC \\
\hline Nueva Loja & Lago Agrio & 4,92 & 4,99 & 5,15 & 4,68 & 5,06 & 4,79 & 4,62 & 4,68 & 4,92 & 5,12 & 5,14 & 5 & 4,91 \\
\hline Lumbaqui & $\begin{array}{c}\text { Gonzalo } \\
\text { Pizarro }\end{array}$ & 4,97 & 4,99 & 5,15 & 5,21 & 5,06 & 4,79 & 4,62 & 4,68 & 4,92 & 5,12 & 5,14 & 5 & 4,91 \\
\hline \begin{tabular}{|c|} 
El \\
Reventador
\end{tabular} & $\begin{array}{c}\text { Gonzalo } \\
\text { Pizarro }\end{array}$ & 4,97 & 4,99 & 5,15 & 5,21 & 5,06 & 4,79 & 4,61 & 4,68 & 4,92 & 5,12 & 5,14 & 5,01 & 4,92 \\
\hline \begin{tabular}{|c|} 
El \\
Reventador
\end{tabular} & $\begin{array}{c}\text { Gonzalo } \\
\text { Pizarro }\end{array}$ & 4,97 & 4,99 & 5,15 & 5,21 & 5,06 & 4,79 & 4,61 & 4,68 & 4,92 & 5,12 & 5,14 & 5,01 & 4,92 \\
\hline \begin{tabular}{|c}
$\begin{array}{c}\text { Gonzalo Diaz } \\
\text { de Pineda }\end{array}$ \\
\end{tabular} & El Chaco & 4,97 & 5 & 5,16 & 5,21 & 5,06 & 4,78 & 4,61 & 4,67 & 4,91 & 5,12 & 5,14 & 5,01 & 4,92 \\
\hline Santa Rosa & El Chaco & 4,97 & 5 & 5,16 & 5,21 & 5,06 & 4,78 & 3,98 & 4,67 & 4,91 & 5,12 & 5,14 & 5,01 & 4,93 \\
\hline $\begin{array}{c}\text { San } \\
\text { Francisco }\end{array}$ & Quijos & 4,31 & 4,34 & 4,48 & 4,52 & 4,38 & 4,14 & 3,98 & 4,04 & 4,26 & 4,44 & 4,46 & 4,35 & 34,3 \\
\hline Papallacta & Quijos & 4,31 & 4,34 & 4,48 & 4,52 & 4,38 & 4,14 & 3,99 & 4,04 & 4,26 & 4,44 & 4,46 & 4,35 & 4,28 \\
\hline Yaruqui & Quito & 4,31 & 4,33 & 4,47 & 4,52 & 4,38 & 4,15 & 4 & 4,05 & 4,26 & 4,44 & 4,46 & 4,35 & 4,27 \\
\hline Nono & Quito & 4,31 & 4,33 & 4,47 & 4,52 & 4,39 & 4,15 & 4 & 4,05 & 4,26 & 4,44 & 4,46 & 4,34 & 4,26 \\
\hline $\begin{array}{c}\text { Pedro } \\
\text { Vicente }\end{array}$ & $\begin{array}{c}\text { Pedro } \\
\text { Vicente }\end{array}$ & 4,97 & 4,99 & 5,15 & 5,21 & 5,06 & 4,79 & 4,62 & 4,68 & 4,92 & 5,12 & 5,14 & 5 & 4,91 \\
\hline Puerto Quito & $\begin{array}{l}\text { Puerto } \\
\text { Quito }\end{array}$ & 4,97 & 4,98 & 5,15 & 5,21 & 5,06 & 4,79 & 4,62 & 4,68 & 4,92 & 5,12 & 5,13 & 5 & 4,91 \\
\hline Rosa Zarate & Quininde & 4,97 & 4,97 & 5,14 & 5,21 & 5,07 & 4,8 & 4,63 & 4,69 & 4,93 & 5,12 & 5,13 & 4,99 & 4,9 \\
\hline Viche & Quininde & 4,97 & 4,96 & 5,14 & 5,21 & 5,07 & 4,81 & 4,64 & 4,7 & 4,93 & 5,13 & 5,12 & 4,98 & 4,88 \\
\hline Vuelta Larga & Esmeraldas & 4,97 & 4,95 & 5,13 & 5,21 & 5,08 & 4,82 & 4,66 & 4,72 & 4,94 & 5,13 & 5,12 & 4,97 & 4,87 \\
\hline
\end{tabular}

Figura 4. ANGSTRÖM-PAGE, promedios mensual y anual de radiación solar para las estaciones meteorológicas del OCP

\section{Análisis Estadístico de las Observaciones en OCP}


Para el cálculo estadístico se toma como población el total de observaciones de radiación solar durante un año calendario (en el horario de 06H00 a 19H00), medidas en las 15 estaciones meteorológicas automáticas instaladas en la ruta del oleoducto de crudos pesados, ubicado en la región norte del Ecuador, tomándose 62780 unidades estadísticas colectadas en el año 2013, alrededor de 4185 observaciones por estación del OCP. Se presentó un faltante de 14 horas de mediciones consecutivas en el día, en 3 estaciones del OCP: (Lumbaqui), en los meses febrero, agosto, septiembre y octubre; Reventador (El Reventador), en el mes de abril. (Santa Rosa), en los meses enero, febrero y marzo, lo que constituyó el $4.65 \%$ de las mediciones por hora. Las 12 estaciones restantes cuentan con el registro completo de horas, días y meses. Posteriormente, se realizó la sumatoria de la irradiancia [W/m2] (observaciones de radiación solar de 06H00 a 19H00) para obtener la irradiación [kWh/m2/día] para cada día del mes, y por estación meteorológica. Para corroborar la calidad de los datos obtenidos se consultan los mantenimientos realizados a las estaciones en el mes de abril 2013 y diciembre 2015 (Fernandez, 2013).

Se calculó la radiación solar global para cada mes del año, utilizando los valores diarios, a través del análisis estadístico para obtener el valor medio observado en superficie horizontal, en el trayecto del OCP. Estos datos corresponden al "mes peor", método desarrollado por Ingeniería Sin Fronteras para el planteamiento de proyectos para aprovechamiento del recurso solar (Fronteras, 1999). Estos resultados se contrastan con la metodología denominada "día tipo". Se calcula la media anual como valor representativo de radiación solar global, estos se relacionan en la figura 5 (OMM, 2008).

\begin{tabular}{|c|c|c|c|c|c|c|c|c|c|c|c|c|c|c|}
\hline \multirow{2}{*}{\multicolumn{3}{|c|}{ OOCPECUADOR }} & \multicolumn{12}{|c|}{ MONTHLY AVERAGEDINSOLATION INCIDENT ON HORIZONTAL SURFACE } \\
\hline & & & \multicolumn{12}{|c|}{$\mathrm{KWh} / \mathrm{m} 2 / \mathrm{day}$} \\
\hline PARROQUIA & CANTON & $\begin{array}{c}\text { ANUAL } \\
\text { AVERAGE }\end{array}$ & JAN & FEB & MAR & APR & MAY & JUN & JUL & AUG & SEP & OCT & NOV & DEC \\
\hline Nueva Loja & Lago Agrio & 3,42 & 3,29 & 2,76 & 3,16 & 3,62 & 2,99 & 2,97 & 3,26 & 3,6 & 4,19 & 4,19 & 3,5 & 3,45 \\
\hline Lumbaqui & $\begin{array}{c}\text { Gonzalo } \\
\text { Pizarro }\end{array}$ & 3,66 & 3,73 & - & 2,77 & 4,3 & 3,47 & 3,55 & 3,16 & - & - & - & 3,72 & 4,51 \\
\hline \begin{tabular}{|c|} 
El \\
Reventador \\
\end{tabular} & $\begin{array}{l}\text { Gonzalo } \\
\text { Pizarro }\end{array}$ & 2,79 & 2,42 & 2,02 & 2,37 & 3,07 & 2,64 & 2,51 & 2,56 & 2,8 & 3,42 & 3,9 & 2,94 & 2,83 \\
\hline \begin{tabular}{|c|} 
El \\
Reventador \\
\end{tabular} & $\begin{array}{l}\text { Gonzalo } \\
\text { Pizarro }\end{array}$ & 2,43 & 2,07 & 1,79 & 2,1 & - & 2,02 & 2,03 & 2,04 & 2,25 & 2,59 & 3,78 & 3,54 & 2,53 \\
\hline $\begin{array}{c}\text { Gonzalo Diaz } \\
\text { de Pineda }\end{array}$ & El Chaco & 3,35 & 2,98 & 2,34 & 3,04 & 3,68 & 3,24 & 3,11 & 2,94 & 3,3 & 3,77 & 4,32 & 4,02 & 3,49 \\
\hline Santa Rosa & El Chaco & 3,32 & - & - & - & 3,7 & 3,27 & 2,89 & 2,57 & 2,69 & 3,57 & 3,96 & 4,05 & 3,18 \\
\hline $\begin{array}{c}\text { San } \\
\text { Francisco }\end{array}$ & Quijos & 3,47 & 3,04 & 2,59 & 3,27 & 3,65 & 3,49 & 3,15 & 3,02 & 3,45 & 3,62 & 4,28 & 4,35 & 3,69 \\
\hline Papallacta & Quijos & 3,46 & 3,4 & 2,79 & 3,07 & 3,63 & 3,65 & 3,04 & 2,8 & 3,29 & 3,68 & 3,66 & 4,47 & 3,64 \\
\hline Yaruqui & Quito & 4,61 & 5,28 & 4,11 & 4,46 & 4,59 & 3,78 & 4,36 & 4,48 & 4,61 & 4,78 & 4,8 & 4,91 & 5,19 \\
\hline Nono & Quito & 2,99 & 2,66 & 2,44 & 2,74 & 2,79 & 2,44 & 3,15 & 3,37 & 3,13 & 3,48 & 3,01 & 3,7 & 3,02 \\
\hline $\begin{array}{l}\text { Pedro } \\
\text { Vicente }\end{array}$ & $\begin{array}{l}\text { Pedro } \\
\text { Vicente }\end{array}$ & 2,54 & 1,85 & 2,2 & 2,62 & 2,8 & 2,36 & 2,48 & 2,95 & 3,18 & 2,7 & 2,59 & 2,44 & 2,25 \\
\hline Puerto Quito & $\begin{array}{l}\text { Puerto } \\
\text { Quito }\end{array}$ & 3,02 & 2,75 & 3,42 & 3,65 & 3,52 & 2,63 & 2,7 & 2,91 & 3,02 & 3 & 2,74 & 2,84 & 3 \\
\hline Rosa Zarate & Quininde & 3,2 & 2,76 & 3,56 & 3,68 & 3,74 & 3,07 & 3,04 & 3,06 & 3,27 & 3,24 & 2,61 & 3,09 & 3,07 \\
\hline Viche & Quininde & 3,22 & 2,51 & 3,53 & 3,45 & 3,59 & 3,07 & 3,05 & 3,05 & 3,5 & 3,47 & 3,05 & 3,31 & 3,04 \\
\hline Vuelta Larga & Esmeraldas & 3,21 & 2,62 & 4,11 & 3,76 & 3,6 & 2,6 & 2,92 & 2,76 & 2,96 & 3,19 & 2,99 & 3,72 & 3,32 \\
\hline
\end{tabular}


Figura 5. Promedios mensual y anual de radiación solar por estación meteorológica automática del OCP

\section{Resultados y Discusión}

Contrastación de Resultados Obtenidos en Zonas de Interés para el Aprovechamiento de la Energía Termo Solar.

La figura 6 muestra el valor medio anual representativo de la radiación solar global, en los 15 sitios de la región norte ecuatoriana, obtenido con cada uno de los cuatro métodos empleados en el apartado 2.2.

\begin{tabular}{|c|c|c|c|c|c|}
\hline \multicolumn{6}{|c|}{ RADIACIÓN GLOBAL ANUAL MEDIA PARA SUPERFICIE HORIZONTAL } \\
\hline & & $\begin{array}{c}\text { Observaciones } \\
\text { en superficie }\end{array}$ & $\begin{array}{c}\text { Observaciones } \\
\text { satélite }\end{array}$ & $\begin{array}{c}\text { Estaciones } \\
\text { programa } \\
\text { informático }\end{array}$ & $\begin{array}{c}\text { Estaciones } \\
\text { modelo } \\
\text { mate mático }\end{array}$ \\
\hline PARROQUIA & PROVINCIA & $\begin{array}{l}\text { Estaciones } \\
\text { Met OCP }\end{array}$ & NASA & CENSOLAR & $\begin{array}{c}\text { ANGSTROM- } \\
\text { PAGE }\end{array}$ \\
\hline Nueva Loja & Sucumbios & 3,42 & 3,76 & 3,64 & 4,92 \\
\hline Lumbaqui & Sucumbios & 3,65 & 4,24 & 3,64 & 4,97 \\
\hline $\begin{array}{c}\text { El } \\
\text { Reventador }\end{array}$ & Sucumbios & 2,79 & 3,88 & 4,8 & 4,97 \\
\hline $\begin{array}{c}\text { El } \\
\text { Reventador }\end{array}$ & Sucumbios & 2,43 & 3,88 & 4,99 & 4,97 \\
\hline $\begin{array}{c}\text { Gonzalo Diaz } \\
\text { de Pineda }\end{array}$ & Napo & 3,35 & 3,88 & 3,44 & 4,97 \\
\hline Santa Rosa & Napo & 3,32 & 3,88 & 3,44 & 4,97 \\
\hline $\begin{array}{c}\text { San } \\
\text { Francisco }\end{array}$ & Napo & 3,47 & 3,88 & 4,01 & 4,31 \\
\hline Papallacta & Napo & 3,46 & 4,25 & 4,01 & 4,31 \\
\hline Yaruqui & Pichincha & 4,61 & 4,25 & 43,44 & 4,31 \\
\hline Nono & Pichincha & 2,99 & 4,25 & 4,99 & 4,31 \\
\hline $\begin{array}{c}\text { Pedro } \\
\text { Vicente }\end{array}$ & Pichincha & 2,54 & 3,96 & 3,64 & 4,97 \\
\hline Puerto Quito & Pichincha & 3,02 & 4,2 & 4,78 & 4,97 \\
\hline Rosa Zarate & Esmeraldas & 3,2 & 4,2 & 4,61 & 4,97 \\
\hline Viche & Esmeraldas & 3,22 & 4,2 & 4,61 & 4,97 \\
\hline Vuelta Larga & Esmeraldas & 3,21 & 4,2 & 3,86 & 4,97 \\
\hline
\end{tabular}

Figura 6. Análisis comparativo de resultados de radiación $(\mathrm{Kwh} / \mathrm{m} 2 /$ día) en la región norte de Ecuador.

\section{Identificación de sitios con mayor potencial de radiación solar en el área de estudio}

La radiación solar global determinada en superficie en el área de estudio permite establecer sitios con potencial para aplicaciones de media y alta temperatura, los cuales se muestran en la figura 7. 


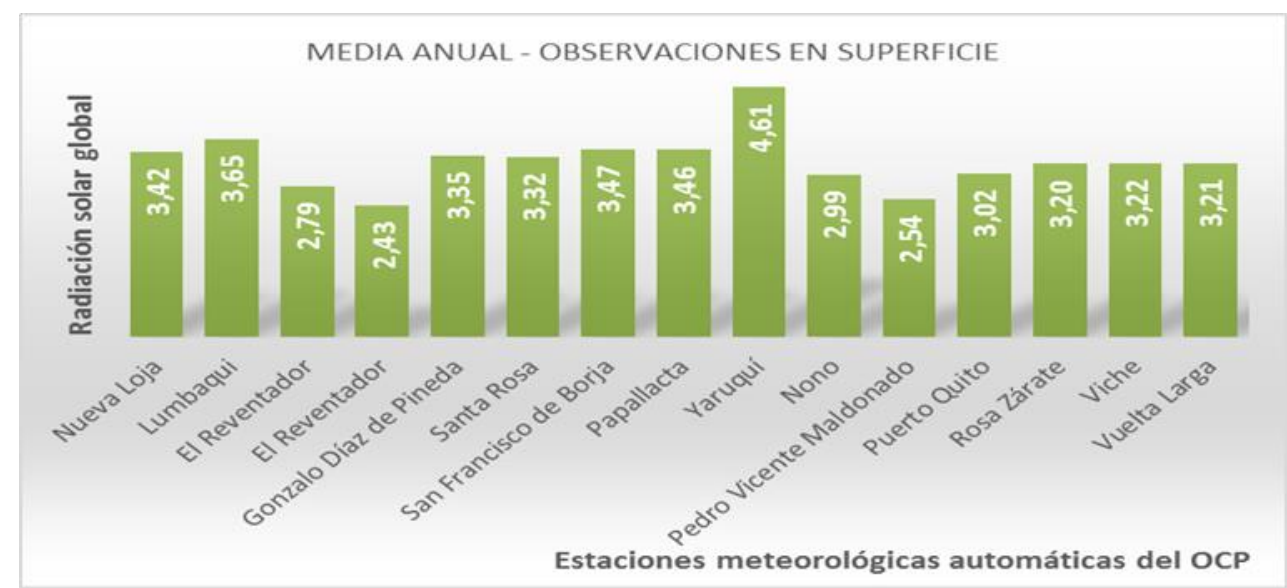

Figura 7. Sitios con potencial para aplicaciones de media y alta temperatura

La figura 8, muestra la representatividad de la radiación solar global determinada en superficie en el área circundante al OCP

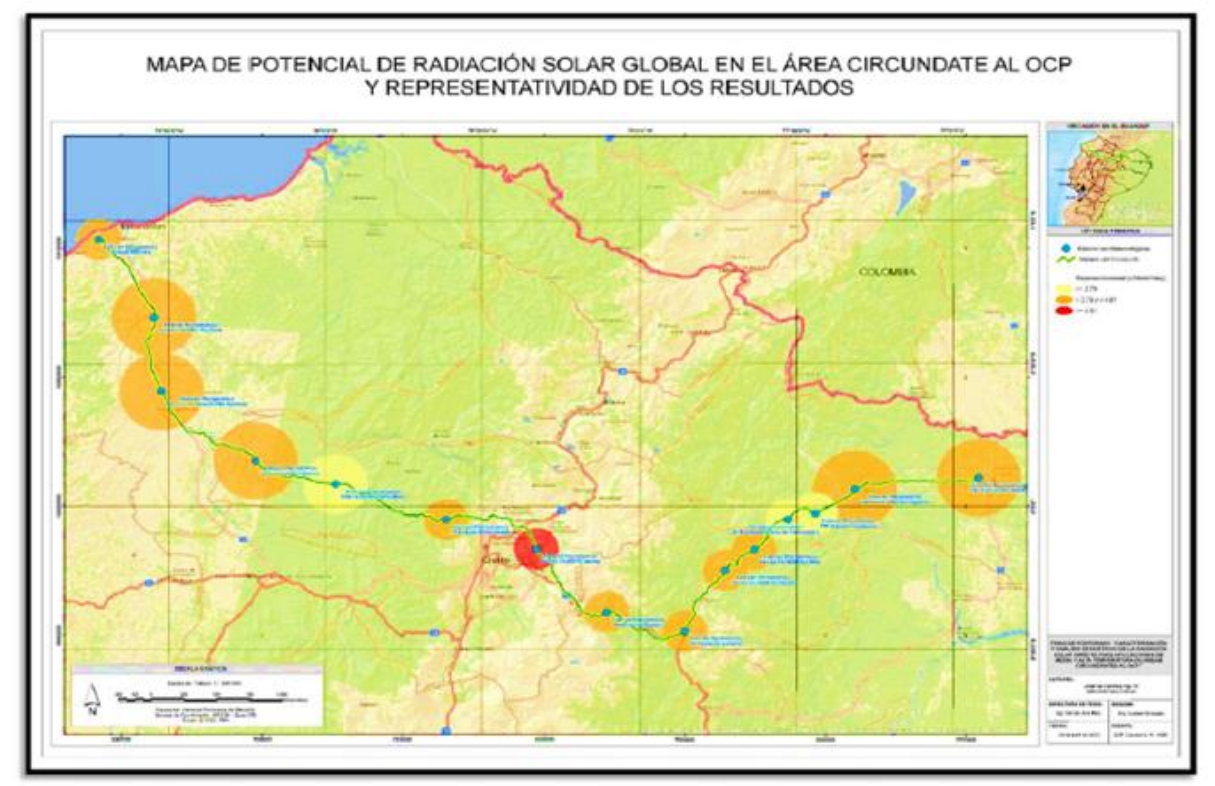

Figura 8. Representatividad de la radiación solar global determinada en superficie en el área circundante.

La radiación solar global determinada a través de los instrumentos calibrados de las estaciones meteorológicas difiere de valores obtenidos por otros métodos, por ser estas netas estimaciones basadas en condiciones climáticas ideales mas no reales, como es el caso de las observaciones en superficie que evidentemente sí tienen influencia de la presencia de variables impredecibles en el entorno como: lluvia, vegetación, edificaciones, etc, que generan microclimas que afectan la medición del recurso solar que llega al área de estudio.

La diferencia entre los valores de radiación obtenidos por software, satélite y modelo matemático, respecto a las mediciones realizadas en superficie, difieren en valores aproximados de $2 \mathrm{kWh} / \mathrm{m} 2 /$ día. 


\section{Conclusiones}

La utilización de los métodos descritos en este documento, tanto modelos matemáticos, imágenes satelitales y programas informáticos, podrían sobredimensionar el diseño de instalaciones, generando falsas expectativas en cuanto al aprovechamiento final del recurso solar.

La evaluación del recurso solar con menor incertidumbre es la observación en superficie a través de estaciones meteorológicas automáticas, en segundo término está la base de datos de la NASA, en tercer lugar el programa informático de CENSOLAR y, finalmente el modelo matemático de Angström-Page.

La comparación entre las observaciones en superficie y los resultados proporcionados por la NASA, muestra variaciones en valores de radiación, los mismos que pueden deberse a que se descarta la existencia de microclimas que pueden ocasionar variaciones en la estimación del recurso.

Los sitios con mayor potencial para posibles aplicaciones de media y alta temperatura son: la parroquia de Yaruquí en Pichincha, Lumbaqui en Sucumbíos, San Francisco de Borja y Papallacta en Napo y Nueva Loja, en ese orden.

Para el dimensionamiento de aplicaciones de media y alta temperatura, tomar en cuenta la diferencia encontrada entre los valores de mediciones en superficie y los obtenidos por modelos de simulación y formulación matemática.

\section{Bibliografía}

Calvache, M., \& Verdugo, J. (14 de 03 de 2011). Diseño y construcción de un sistema móvil de iluminación con capacidad de 300 WP utilizando energía solar para el Laboratorio de Energías Renovables (Tesis de pre grado Universidad de las Fuerzas Armadas Espe, Quito, Ecuador). Quito, Pichincha, Ecuador. Recuperado el 28 de 03 de 2015, de http://repositorio.espe.edu.ec/handle/21000/2860?mode=full

Cañada, L. (1983). El mapa bioclimático del Ecuador. Texas: Banco Central del Ecuador.

Fernández, F. S. (2013). Reporte de visita OCP- Estaciones y Válvulas. Quito.

Fronteras, I. s. (1999). Energía Solar Fotovoltaica y cooperación al desarrollo. Recuperado el 25 de 02 de 2015, de eurosur: http://www.eurosur.org/medioambiente/esf/

INAMHI. (s.f.). INAMHI. Obtenido de http://www.serviciometeorologico.gob.ec/

Jutglar, L. (2004). Energía Solar. Barcelona, España: Grupo Planeta (GBS). Recuperado el 18 de 11 de 2014, de https://books.google.es/books/about/Energ\%C3\%ADa_solar.html?id=10qPPTGMRUYC

Meteoro, C. (2015). Reporte de Visita OCP- Estaciones y Valvulas. Quito. 
Núñez, S., \& Gentile, E. (2004). Universidad de Buenos Aires. Recuperado el 28 de 03 de 2015, de uba: http://www.filo.uba.ar/contenidos/carreras/geografia/catedras/climatologia/sit

Núñez, S., \& Gentile, E. (2004). La observación meteorológica. Recuperado el 28 de 03 de 2015, de Universidad de Buenos Aires: http://www.filo.uba.ar/contenidos/carreras/geografia/catedras/climatologia/sitio/obsmet20 04.pdf

OCP. (2012). Informe de Análisi de Estaciones Metereológicas y Clima OCP Geosuelos Cía. Ltda Consultores Ingeniería Quito. Quito.

OMM. (2008). Organizacion Metereologica Mundial. (S. Géneve, Editor) Recuperado el 25 de 03 de 2015, de wmo: https://www.wmo.int/pages/index_es.html

OpenCourseWare, UNIA. (s.f.). Modelos para estimar la Radiacion Solar. Recuperado el 23 de 07 de 2014, de Universidad Internacional de Andalucía: http//ocw.unia.es/cienciastecnologicas/caracterizacion-y-evaluacion-de-la-radiacion-solar/tema-3

Orellana, G. J., \& Orellana Samaniego, M. L. (Febrero de 2015). Estimación de la Radiación Solar Cantón Cuenca Mediante la Aplicación del Modelo Bristow y Campbell (Tesis de pregrado de la Universidad Politécnica Salesiana, Cuenca, Ecuador). Cuenca, Azuay, Ecuador. $\begin{array}{lllllll}\text { Recuperado } & \text { el } & 15 & \text { de } & 04 & \text { de } & 2015,\end{array}$ http://dspace.ups.edu.ec/bitstream/123456789/8428/1/UPS-CT004934.pdf

Prando, R. (2014). Energías renovables en la industria de procesos. Obtenido de Universidad de la República - Uruguay: http://www.fing.edu.uy/iq/cursos/qica/industria/EERR-Solar.pdf

Suntaxi, J. (29 de febrero de 2014). Caracterización energética del sistema hidroconvencion de la luz solar (Tesis de pregrado de la Universidad de las Fuerzas Armadas Espe, Quito, Ecuador).

Quito, Pichincha, Ecuador: Tesis de maestría, Departamento de la Energía y Mecánica de la Universidad de las Fuerzas Armadas- Espe.

Velasco Roldan, L., Goyos Pérez, L., Freire Amores, L., \& Ibarra, A. (2015). Potencial de aprovechamiento de la biomasa vegetal como aislamiento en climas extremos del Ecuador. Enfoque UTE, 2. 